Rev. Inst. Flor. v. 27 n. 2 p. $183-189$ dez. 2015

http://dx.doi.org/10.4322/rif.2015.015

ISSN impresso 0103-2674/on-line 2178-5031

\title{
OCORRÊNCIA E DANOS DE TÉRMITAS NA MADEIRA DE Acacia mearnsii (FABACEAE) EM DOIS CAMPOS DE APODRECIMENTO (NOTA CIENTÍFICA) ${ }^{1}$
}

\section{OCCURRENCE AND DAMAGE OF TERMITES IN WOOD OF Acacia mearnsii (FABACEAE) IN TWO DECAYING FIELDS (SCIENTIFIC NOTE)}

\author{
Amanda Grassmann da SILVEIRA ${ }^{2,4}$; Elio José SANTINI ${ }^{2}$; Rômulo TREVISAN³; \\ Luciano Campos CANCIAN²; Lilian Gonçalves MARIANO²
}

\begin{abstract}
RESUMO - Entender a relação entre praga e ambiente é base para um adequado gerenciamento no setor madeireiro, assim como a busca por informações sobre a qualidade da madeira de espécies exóticas, que são alternativas para suprir a sua demanda. Dessa maneira, o trabalho objetivou verificar a ocorrência e danos de térmitas na madeira de Acacia mearnsii De Wild. (Fabaceae) em dois campos de apodrecimento. O experimento foi realizado em área de pastagem e de mata nativa. A avaliação foi realizada de forma visual, com atribuição de notas de acordo com o estado fitossanitário de cada peça. Os moirões foram divididos em: parte subterrânea (contato com o solo) e aérea (parte do moirão situada acima do solo). Térmitas atacaram somente peças em mata nativa, não ocorrendo danos dos insetos em área de pastagem. A influência dos organismos foi determinada através do Índice de Deterioração - I.D., onde das sete peças da parte subterrânea presentes em mata nativa, quatro sofreram ataque moderado (I.D. $=70$ ), uma apresentou ataque intenso (I.D. $=40$ ), e duas, ataque leve ou superficial (I.D. $=90$ ). Quando analisada somente a parte aérea, verificaram-se poucos danos, sendo atribuído I.D. geral de 90, ou seja, ataque leve. Os resultados foram associados às características edafoclimáticas de cada ambiente, sendo que em mata nativa o desenvolvimento de térmitas foi melhor devido à menor temperatura ambiente, maior umidade relativa do ar, menor movimentação de ar, maior porosidade e menor densidade do solo.
\end{abstract}

Palavras-chave: deterioração; durabilidade natural; monitoramento; organismos xilófagos; Isoptera.

\footnotetext{
'Recebido para análise em 23.04.15. Aceito para publicação em 29.10.15.

${ }^{2}$ Universidade Federal de Santa Maria, campus Universitário Dr. José Mariano da Rocha Filho, Av. Roraima, 1000, 97105-900 Santa Maria, RS, Brasil. ${ }^{3}$ Universidade Federal de Santa Maria, campus de Frederico Westphalen, Linha Sete de Setembro, s/n, BR386 KM40, 98400-000, Frederico Westphalen, RS, Brasil.

${ }^{4}$ Autor para correspondência: Amanda Grassmann da Silveira - amandagrassmann@gmail.com
} 


\begin{abstract}
Understand the relationship between plague and environment is the basis for an appropriate management in timber industry, as well as the search for information on wood quality of exotic species, which are alternatives to its demand. In this way, the work aimed to check the occurrence and damage of termites in wood of Acacia mearnsii De Wild. (Fabaceae) in two rotting field. The experiment was accomplished in pasture area and native forest. The evaluation was performed visually, with assignment of notes according to the phytosanitary state of each piece. Round fence posts were divided into: underground part (contact with soil) and shoot (part of round fence posts located above the ground). Termites have attacked only parts in native forest, no occurring insect damage in the pasture area. The influence of the organisms was determined by the rate of deterioration - I.D. wherein the seven pieces of underground part present in native forest, four suffered moderate attack four suffered moderate attack (I.D. $=70)$, one presented intense attack (I.D. $=40)$ and and two presented light or superficial attacks (I.D. $=90)$. There was little damage when analyzed only the aerial part, being assigned I.D. 90, general gentle attack. The results were associated to soil and climate characteristics in each environment, and in the development of native forest termites was better due to the lower ambient temperature, higher relative humidity, less air movement, higher porosity and lower bulk density of the soil.
\end{abstract}

Keywords: deterioration; natural durability; monitoring; xylophagous organisms; Isoptera.

\section{INTRODUÇÃO}

A durabilidade natural da madeira permite avaliar a vida média útil e a susceptibilidade a organismos xilófagos. Assim, ensaios de campo com material em contato com o solo possibilitam a classificação da sua durabilidade, recomendando-se, ou não, o uso em ambientes externos (Jesus et al., 1998). Os ensaios de campo, embora constituam métodos simples, reproduzem fielmente as condições reais de serviço, nas quais peças de diferentes espécies e com dimensões preestabelecidas são expostas às intempéries e ao contato com o solo e avaliadas quanto ao grau de deterioração (Fosco Mucci et al., 1992).

A madeira está sujeita a danos por insetos desde a árvore viva até sua utilização, e as principais ordens encontradas são Isoptera, Coleoptera e, com menor importância, Hymenoptera (Eaton e Hale, 1993). Os cupins são considerados severos agentes destruidores, porém, estes organismos são importantes para a manutenção da dinâmica dos processos de decomposição e para os fluxos de carbono e nutrientes nas florestas tropicais (Bandeira e Vasconcellos, 2002).

Os cupins ou térmitas são insetos sociais que vivem em colônias. Sendo mastigadores, têm a celulose como seu principal alimento, a qual pode vir de uma grande variedade de produtos. O material madeireiro, devido ao seu alto teor de celulose, é o alimento preferido por um variado número de espécies (Oliveira e Lepage, 1986).
De modo geral, as espécies de cupins podem ser divididas em subterrâneas e de madeira seca (Richardson, 1993). Em estudos de degradação de madeira, os térmitas são classificados conforme o tipo de ninho que constroem, sendo dada ênfase aos cupins subterrâneos, tanto pela dificuldade no controle quanto pela proliferação maciça em ambientes urbanos (Santini, 1988). Segundo o mesmo autor, os insetos atacam preferencialmente madeiras em contato com o solo, sendo facilmente perceptíveis pelas suas típicas galerias ascendentes.

A construção de ninhos e sistemas de túneis subterrâneos, realizados por térmitas, causam modificações na estrutura do solo, promovendo aumento de porosidade e aeração (Wood e Sands, 1978). A colônia desses cupins, de acordo com Cavalcante (1985), é composta de milhares de indivíduos, localizada no solo, de onde parte para o ataque. A ocorrência desse tipo de cupim é denunciada pela presença de túneis de terra nas peças.

O comportamento de uma mesma madeira pode variar de acordo com o ambiente, pois este apresenta características específicas de umidade, insolação, aeração, temperatura e presença de organismos xilófagos. Tais fatores atuam conjuntamente sobre a madeira, determinando sua durabilidade natural (Mendes e Alves, 1988). 
A decomposição de materiais biológicos está relacionada a uma série de fatores bióticos e abióticos, como as condições climáticas, composição da comunidade decompositora, afinidade com o substrato e as características do material exposto. Esses elementos, quando correlacionados, fecham o ciclo da deterioração, sendo ainda preciso acrescentar a ação do sol sobre a madeira, que gera uma variação de temperatura e nas tensões criadas pelo umedecimento e secagem, tornando-se contribuintes no processo (Swift et al., 1979).

A exposição constante à umidade e $\mathrm{o}$ contato direto com o solo tornam a madeira suscetível à rápida deterioração, normalmente ocasionada por fungos e térmitas subterrâneos. Em contraponto, nos ambientes mais secos, o risco de deterioração biológica é menor, e quando ocorre, é caracterizada por ataque de cupins de madeira seca e brocas (Barillari, 2002). Devido a isso, destaca-se a importância em contrastar áreas que apresentem características distintas, permitindo a comparação entre resultados e a busca por soluções mais adequadas, de acordo com os problemas específicos encontrados em cada ambiente.

Quando se trabalha com dois ambientes de características distintas, encontra-se a necessidade de compreender a influência do ambiente no processo de decomposição da madeira, pois estas áreas podem apresentar diferentes níveis de apodrecimento, fato que pode ser explicado por particularidades da própria espécie ou pelas diferentes variáveis climáticas envolvidas. O objetivo deste trabalho foi verificar a ocorrência e os danos de térmitas na madeira de Acacia mearnsii De Wild. em dois campos de apodrecimento.

\section{MATERIAL E MÉTODOS}

O trabalho foi realizado no campo experimental da Universidade Federal de Santa Maria, Campus de Frederico Westphalen, no Médio Alto Uruguai - Rio Grande do Sul, com latitude $27^{\circ} 21^{\prime} \mathrm{S}$ e longitude $53^{\circ} 23^{\prime} \mathrm{O}$. De acordo com a classificação de Köppen (1948), o clima da região é do tipo Cfa - temperado chuvoso, com precipitação média anual elevada, geralmente entre 1.800 e $2.100 \mathrm{~mm}$, bem distribuída ao longo do ano, e subtropical do ponto de vista térmico.

Sete árvores de Acacia mearnsii, com 8 anos de idade, foram abatidas e 14 moirões selecionados. Dois campos de apodrecimento foram formados (sete moirões cada), em duas áreas distintas. O primeiro, em área de pastagem (ambiente 1), e o segundo, dentro de um fragmento de vegetação de Floresta Estacional Decidual (ambiente 2), acompanhados por um período de 12 meses.

Um termo-higrômetro foi utilizado para leitura da temperatura e umidade relativa das áreas, além da coleta de cilindros de solo indeformados para avaliação da porosidade total e da densidade do solo nas áreas de estudo. Para estas avaliações, as amostras foram encaminhadas para o Laboratório de Física do Solo da própria Universidade. Posteriormente, as médias das variáveis foram analisadas estatisticamente pelo teste de Tukey ao nível de $5 \%$ de probabilidade.

$\mathrm{Na}$ avaliação visual das amostras de madeira, as mesmas foram divididas em parte subterrânea ( $30 \mathrm{~cm}$ abaixo da linha de afloramento) e aérea (120 $\mathrm{cm}$ acima da linha de afloramento). A parte aérea do material foi avaliada com amostras ainda em campo, com atribuição de notas de acordo com o estado fitossanitário da peça, considerando apenas o ataque de Isoptera (Lepage, 1970) (Tabela 1). A seguir, os moirões foram retirados e a avaliação da parte subterrânea foi realizada com o mesmo procedimento.

Tabela 1. Classificação do nível de deterioração das madeiras por térmitas (Lepage, 1970).

Table 1. Classification of wood deterioration level by termites (Lepage, 1970).

\begin{tabular}{lcc}
\hline Estado de Sanidade & Nota & Índice de Deterioração \\
\hline Sadio, nenhum ataque & 0 & 100 \\
Ataque leve ou superficial de térmitas & 1 & 90 \\
Ataque evidente, mas moderado de térmitas & 2 & 70 \\
Apodrecimento intenso ou ataque interno de térmitas & 3 & 40 \\
Quebra, perda quase total de resistência & 4 & 0 \\
\hline
\end{tabular}


Os moirões foram retirados e com o auxílio de um machado as peças foram cortadas para facilitar a verificação dos danos causados pelos insetos (Figura 1). O índice de deterioração foi admitido para estabelecer o estado médio de vida útil de cada amostra dentro de cada campo, e, em seguida, estes foram somados e o resultado foi dividido pelo número de amostras (sete), obtendo-se o valor médio (Trevisan, 2006).

A observação dos moirões atacados foi realizada de maneira visual, sendo contabilizado o número de peças atacadas em cada campo, para posterior análise dos dados e contrastes de resultados, a fim de verificar a diferença entre os campos.

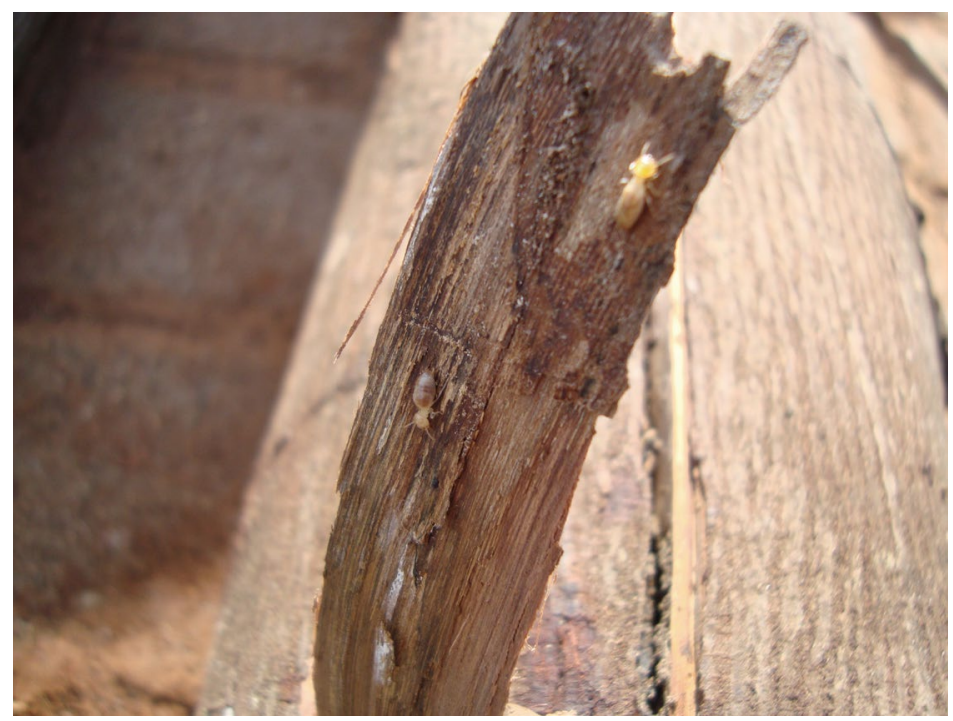

Figura 1. Ataque de cupim em tora de Acacia mearnsii.

Figure 1. Verification of the attack by termites in Acacia mearnsii.

\section{RESULTADOS E DISCUSSÃO}

O levantamento das características edafoclimáticas das diferentes áreas está expresso na Tabela 2. Os resultados demonstram que os ambientes são diferentes, de modo que o ambiente 1 possui maior temperatura e maior densidade do solo, enquanto o ambiente 2 se mostra mais úmido e com solo com maior porosidade total.

Após 12 meses de exposição nos campos de apodrecimento, apenas no ambiente 1 foi verificada a presença de térmitas do gênero Neocapritermes, pertencentes à família Termitidae.
Esses organismos são endêmicos da América do Sul e Central, onde já foram confirmadas 14 espécies, tendo uma ampla distribuição no Brasil (Krishna e Araujo, 1968). Conforme os mesmos autores, esse gênero vive em ninhos epígeos e/ou subterrâneos, com galerias irregulares e são essencialmente geófagos. Esse hábito alimentar está associado ao consumo de material altamente heterogêneo, incluindo desde matéria orgânica, tecidos vegetais fragmentados, paredes celulares lignificadas, xilema velho e seus subprodutos, até itens de alto valor nutricional (Brauman et al., 2000).

Tabela 2. Análise das variáveis edafoclimáticas nos diferentes ambientes.

Table 2. Analysis of the soil and climate variables in different environments.

\begin{tabular}{ccccc}
\hline Áreas & Temperatura & Umidade Relativa & Densidade do Solo & Porosidade Total \\
\hline Floresta (ambiente 2) & $17^{\circ} \mathrm{b}^{*}$ & $83,7 \% \mathrm{a}$ & $0,846 \mathrm{a}$ & $68,1 \mathrm{a}$ \\
Campo aberto (ambiente 1) & $18,9^{\circ} \mathrm{a}$ & $73,9 \% \mathrm{~b}$ & $1,191 \mathrm{~b}$ & $55,0 \mathrm{~b}$ \\
\hline
\end{tabular}

*Médias seguidas pela mesma letra na coluna não diferem estatisticamente entre si pelo teste Tukey ao nível de $5 \%$ de probabilidade de erro.

* Means followed by the same letter in the column do not differ statistically from each other at $5 \%$ probability by Tukey test. 
$\mathrm{Na}$ avaliação visual realizada na parte aérea do material em mata nativa (Figura 2), foi verificado, de forma geral, um ataque leve ou superficial (I.D. $=90)$ das térmitas, enquanto o situado em área de pastagem apresentou danos associados somente a fatores abióticos, e nenhum dano visível referente aos insetos $($ I.D. $=100)$. A pouca incidência, ou até mesmo a ausência de danos nas partes aéreas das peças, se deve ao fato de as mesmas não estarem em contato direto com o solo, dificultando o ataque dos organismos.

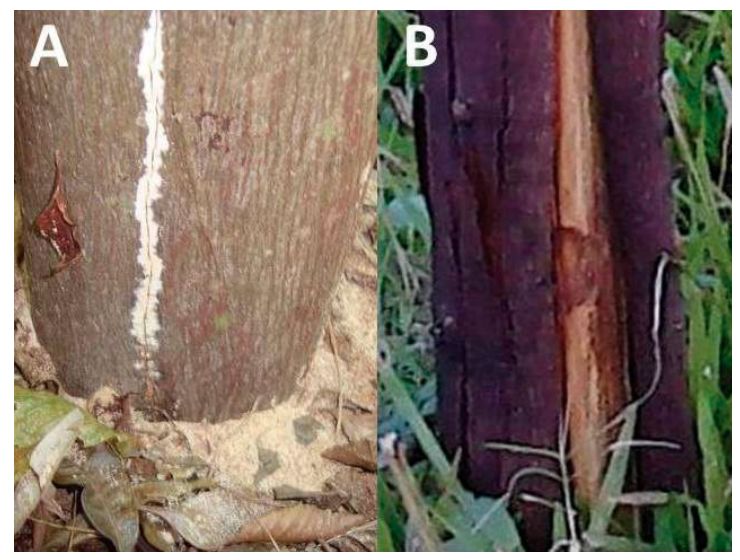

Figura 2. Avaliação visual da parte aérea de toras de Acacia mearnsii: (A) rachadura e serragem, indicativo de cupim no ambiente de mata nativa; (B) danos referentes a fatores abióticos sem indicativo de cupim (área de pastagem).

Figure 2. Visual assessment of Acacia mearnsii round fence posts: (A) crack and sawdust, termite indicative in bushland environment; (B) damage related to abiotic factors with no indication of termites (pasture area).

Quatro amostras da parte subterrânea (Figura 3), presentes em mata nativa, sofreram ataque moderado (I.D. $=70$ ), uma apresentou ataque intenso (I.D. $=40$ ), e duas apresentaram ataque leve ou superficial (I.D. $=90$ ), enquanto no ambiente 1 , os moirões não apresentaram ataques de cupins (I.D. $=100)$.
Moirões presentes no ambiente 2 foram danificados pelos cupins e, em algumas peças, estes se mostraram visualmente expressivos, enquanto no ambiente 1 não foi encontrado nenhum dano que fosse possível associar a estes organismos, fato explicado por não haver nenhum registro da presença do inseto nas amostras provenientes do ambiente 1 .

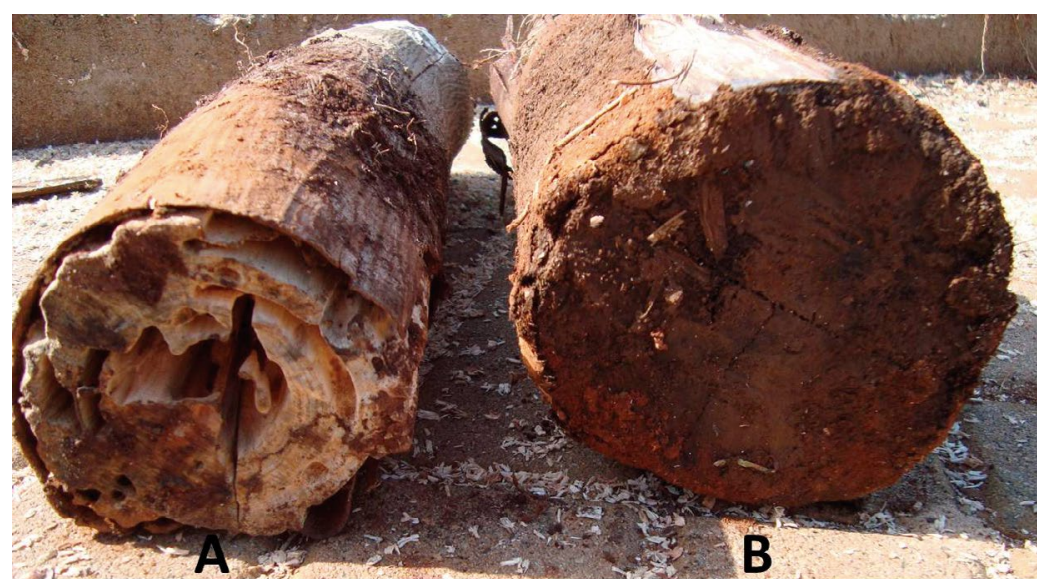

Figura 3. Amostras da parte subterrânea de moirões de Acacia mearnsii: (A) danos causados por cupins em amostras provenientes de dentro da mata nativa; (B) nenhum ataque nas amostras provenientes em área de pastagem.

Figure 3. Samples of the underground part of Acacia mearnsii round fence posts: (A) termite damage in samples from within the native forest, and (B) no attack on samples from pasture area. 
A menor variação de temperatura no ambiente 2, (máxima de $26,7^{\circ} \mathrm{C}$ e mínima de $0,5^{\circ} \mathrm{C}$ ), associada a maior umidade relativa $(83,7 \%)$, pode ter influenciado positivamente na maior ocorrência dos insetos. $\mathrm{O}$ ambiente 1 registrou temperaturas máximas e mínimas de $28,2{ }^{\circ} \mathrm{C}$ e $0,6{ }^{\circ} \mathrm{C}$, respectivamente, com $73,9 \%$ de UR. Essa capacidade de desenvolvimento no ambiente 2 está relacionada à menor temperatura e menor movimentação do ar, que favorece uma melhor estabilização das condições consideradas adequadas à presença dos insetos xilófagos (Seitz, 1976).

Outro fator determinante foi a densidade do solo, que apresentou valores inferiores dentro da mata nativa, com média de $0,84 \mathrm{Mg} \mathrm{m}^{-3}$, enquanto a área de pastagem obteve uma média de $1,19 \mathrm{Mg} \mathrm{m}^{-3}$. Solos com baixa densidade são mais propensos ao ataque de térmitas, devido à maior capacidade de absorção de água (Rocha, 2001). Isso se deve ao fato de os cupins subterrâneos não possuem revestimento de quitina, conferindo-lhes, deste modo, pouca resistência à baixa umidade do solo (Mendes e Alves, 1988). $\mathrm{O}$ solo do ambiente 2 apresentou porosidade total de 68,1\%, possibilitando, deste modo, maior aeração, permitindo maior presença de oxigênio para os indivíduos se desenvolverem. No ambiente 1 , o solo apresentou valores menores de porosidade total, com valor médio de 55\%, tornando o ambiente menos favorável para os organismos, segundo as características descritas pelos autores.

A ocorrência de Isoptera em mata nativa pode ser explicada pela menor incidência de vento, ocasionada pela presença das árvores. Com a fermentação da madeira, ocorre liberação de compostos voláteis (etanólicos) e, supõe-se, que os mesmos fiquem concentrados no próprio local, criando um ambiente atrativo aos insetos. No ambiente, a maior circulação de ar dispersa o odor oriundo da fermentação da madeira e, por conseguinte, reduz o efeito atrativo sobre os insetos (Trevisan, 2006).

A preferência do ataque em mata nativa ainda pode ser explicada sob outro ponto de vista, pois, quando os organismos atacam a madeira e esta se mostra apropriada, a mesma sofre um ataque em massa de outros organismos xilófagos, ocasionado pela síntese e liberação de elementos químicos, que acabam se concentrando no local, devido a menor intensidade de ventos (Lunz, 2001).
Essas substâncias atuam na agregação e no aumento da reprodução, explicando, deste modo, a maior ocorrência de Isoptera nesse ambiente.

\section{CONCLUSÃO}

As características edafoclimáticas foram mais adequadas ao desenvolvimento de Isoptera em mata nativa, com presença e maior grau de severidade destes organismos em todas as peças testadas no ambiente. Na área de pastagem, não foi verificada a presença de cupins, sendo que os danos nas amostras foram causados principalmente por agentes abióticos.

\section{REFERÊNCIAS BIBLIOGRÁFICAS}

BANDEIRA, A.G.; VASCONCELlOS, A. A quantitative survey of termites in a gradient of disturbed highland forest in Northeastern Brazil (Isoptera). Sociobiology, v. 39, p. 429-439, 2002.

\section{BARILlARI, C. T. Durabilidade da madeira} do gênero Pinus tratada com preservantes: avaliação em campo de apodrecimento. 2002. 68 f. Dissertação (Mestrado em Recursos Florestais) Escola Superior de Agricultura "Luiz de Queiroz", Universidade de São Paulo, Piracicaba.

BRAUMAN, A.; BIGNELL D.E.; TAYASU, I. Soil-feeding termites: biology, microbial associations and digestive mechanisms. In: ABE, T.; BIGNELL, D.E.; HIGASHI, M. (Ed.). Termites: evolution, sociality, symbioses, ecology. Dordrecht: Kluwer Academic Publishers, 2000. p. 233-259.

CAVALCANTE, M.S. Métodos para aumentar a durabilidade da madeira. Boletim da ABPM, n. 36, p. 159-170, 1985.

EATON, R.A.; HALE, M.D.C. Wood: decay, pests and protection. London: Chapman \& Hall, $1993.546 \mathrm{p}$.

FOSCO MUCCI, E.S.; LOPEZ, G.A.; MONTAGNA, R.G. Durabilidade natural de madeiras em contato com o solo - IV. In: CONGRESSO NACIONAL SOBRE ESSÊNCIAS NATIVAS, 2., 1992, São Paulo. Anais... São Paulo: UNIPRESS, 1992. p. 558-563. (Rev. Inst. Flor., v. 4, pt. 2, 1992). 
SILVEIRA, A.G. da et al. Ocorrência e danos de térmitas na madeira de Acacia mearnsii

JESUS, M.A.; MORAIS, J.W.; ABREU, R.L.S. Durabilidade natural de 46 espécies de madeira amazônica em contato com o solo em ambiente florestal. Scientia Forestalis, n. 54, p. 81-92, 1998.

KÖPPEN, W. Climatología: con un estudio de los climas de la Tierra. Mexico: Fondo de Cultura Economica, 1948. 466 p.

KRISHNA, K.; ARAUJO, R.L. A revision of the neotropical termite genus Neocapritermes (Isoptera, Termttidae, Teirmitinae). Bulletin of the American Museum of Natural History, v. 138, p. 83-130, 1968.

LEPAGE, E.S. Método sugerido pela IUFRO para ensaios de campo com estacas de madeira. Preservação de Madeiras, v. 1, n. 4, p. 205-216, 1970.

LUNZ, A.M. Degradação da madeira de seis espécies arbóreas causada por Scolytidae (Coleoptera). 2001. 160 f. Dissertação (Mestrado em Ciências Ambientais e Florestais) - Universidade Federal Rural do Rio de Janeiro, Rio de Janeiro.

MENDES, A.S.; ALVES, M.V.S.A Degradação da madeira e sua preservação. Brasília, DF: Instituto Brasileiro de Desenvolvimento Florestal, 1988. 56 p.

OLIVEIRA, A.M.F.; LEPAGE, E.S. Controle de qualidade. In: LEPAGE, E.S. (Coord.). Manual de preservação de madeiras. São Paulo: IPT, SICCT, 1986. p. 99-279.

RICHARDSON, B.A. Wood preservation. 2. ed. London: E \& FN SPON, 1993. 226 p.

ROCHA, M.P. Biodegradação e preservação da madeira. Curitiba: FUPEF, 2001. 94 p. (Série Didática, 1).

SANTINI, E.J. Biodeterioração e preservação da madeira. Santa Maria: UFSM/CEPEF/FATEC, 1988. 125 p.

SEITZ, R. A. Estudo da variação da radiação solar, temperatura e umidade relativa do ar no interior de uma mata de Araucaria angustifolia em relação ao terreno livre. Revista Floresta, v. 7, p. 36-45, 1976.
SWIFT, M.J.; HEAL, D.W.; ANDERSON, J.M. Studies in ecology - decomposition in terrestrial and aquatic ecosystems. Oxford: Blackwell, 1979. $371 \mathrm{p}$.

TREVISAN, H. Degradação natural de toras e sua influência nas propriedades físicas e mecânicas da madeira de cinco espécies florestais. 2006. 56 f. Dissertação (Mestrado em Ciências Ambientais e Florestais) - Universidade Federal Rural do Rio de Janeiro, Rio de Janeiro.

WOOD, T.G.; SANDS, W.A. The role of termites in ecosystems. In: BRIAN, M.V. (Ed.). Production ecology of ants and termites. Cambridge: Cambridge University Press, 1978. p. 245-292. 